\title{
Organização da atenção em saúde bucal pelo Programa Saúde nas Escolas: levantamento de necessidades
}

\author{
Erika Guidetti*; Magda Moura de Almeida**. \\ * Residente em Saúde da Família pela Escola de Saúde Pública do Ceará. \\ ** Professora Assistente do curso de Medicina da Universidade de Fortaleza \\ (UNIFOR).
}

\section{RESUMO}

O objetivo do presente trabalho foi realizar o levantamento de necessidades bucais em estudantes do município de Fortaleza no bairro Passaré. O estudo foi descritivo transversal em uma escola pública. A primeira etapa consistiu na instrução de higiene oral e escovação supervisionada com dentifrícios fluoretados em todos os alunos. Em seguida em um ambiente com luz natural foi realizado o exame clínico. De acordo com as características bucais, foi preenchida uma ficha com os seguintes códigos: 0 - sem necessidades, 1 sangramento gengival, 2 - atividade de cárie até 3 cavidades/mancha branca, 3 atividade de cárie até 4 cavidades ou mais e 4 - raiz residual. O processamento dos dados foi realizado em no software SPSS $\AA$ 16. Foi examinado um total de 186 estudantes, distribuídos em 12 turmas, desde pré-escolares até escolares. De todos os estudantes, 55,4\% apresentaram alguma alteração bucal. Dos pré-escolares, 43,4\% possuíam atividade de cárie, sangramento ou raiz residual, enquanto nos escolares essa frequência foi de 57,6\%. A prevalência encontrada nessa comunidade encontra-se abaixo da prevalência nacional e estadual. Inferindo provavelmente o bom trabalho preventivo dos cirurgiões dentistas deste território.
Descritores:Levantamento epidemiológico. Prevalência. Estudantes. Saúde bucal

\section{INTRODUÇÃO}

Anteriormente à criação do SUS, a assistência odontológica pública, a exemplo de outros setores da saúde, atendia somente os trabalhadores contribuintes ao Instituto Nacional de Assistência Médica da Previdência Social (INAMPS), por meio de convênios e credenciamentos do Estado com o setor privado ${ }^{17}$.

Somente com a promulgação da Constituição Federal de 1988, o acesso universal da população aos serviços de saúde foi garantido legalmente. A criação do SUS representou o "rompimento com a lógica da assistência vinculada aos interesses do patronato e com a ação curativista"1 $^{17}$.

A Odontologia Integral ou o Incremental Modificado enfatiza a prevenção, reconhece a cárie como doença infecto-contagiosa, institui o retorno programado para manutenção preventiva e utiliza a idéia de equipe odontológica, formada pelo cirurgião-dentista, pelo técnico de higienização dentária e pelo auxiliar de consultório dentário ${ }^{17}$.

Ele adapta sua estrutura organizativa à doutrina do SUS e apresenta como 
característica o controle epidemiológico da doença cárie através da utilização de métodos preventivos. Somente após controlada a doença, inicia-se o tratamento restaurador definitivo, reabilitação oral e ampliação da cobertura populacional ${ }^{17}$.

Um novo modelo de atenção à saúde de acordo com os Cadernos de Atenção Básica - Saúde na Escola ${ }^{5}$, foi adotado a partir da intersetorialidade entre a escola e o serviço de saúde, visando o fortalecimento de prática de produção e promoção da saúde, consideração do espaço escolar como ambiente potencial para a produção de práticas de saúde. As ações nesses campos têm mútuas repercussões e, assim sendo, a construção de ações integradas é condição indispensável para atualizar e renovar, de forma permanente, os significados da educação e da saúde, com vistas à integralidade.

O Programa Saúde na Escola (PSE), política intersetorial da Saúde e da Educação, foi instituído em 2007. As políticas de saúde e educação voltadas às crianças, adolescentes, jovens e adultos da educação pública brasileira se unem para promover saúde e educação integral. A articulação entre Escola e Rede Básica de Saúde é a base do Programa Saúde na Escola. O PSE é uma estratégia de integração da saúde e educação para o desenvolvimento da cidadania e da qualificação das políticas públicas brasileiras $^{13}$.

O PET-Saúde é uma das estratégias de indução a mudanças curriculares nos cursos de graduação na área da saúde, e permite ao acadêmico de odontologia realizar imersão neste tipo de prática ${ }^{10}$.

Este projeto foi fruto de parceria do PET-Saúde/UNIFOR com atividades do Projeto Saúde do Escolar (PSE), para avaliação das condições de saúde das crianças, adolescentes e jovens que estão na escola pública, com o objetivo de realizar o levantamento de necessidades bucais em estudantes do município de Fortaleza no bairro Passaré.

Mesmo com os indicadores epidemiológicos registrando grandes declínios, a cárie dental ainda representa uma das morbidades de maior importância na saúde pública do Brasil ${ }^{6}$.

A saúde bucal no Brasil ainda apresenta precariedade e merece atenção de acordo com estudos epidemiológicos. As crianças possuem um dos mais altos índices de exodontias prematuras. Lesões de cárie extensas são fatores que podem desencadear mal-oclusões ${ }^{13}$. Portanto, pretende-se que os dados provenientes desse estudo possam tornar-se indicadores que permitam a avaliação das políticas públicas direcionadas a saúde infantil e saúde bucal na região.

\section{MATERIAL E MÉTODOS}

Foi realizado um estudo descritivo transversal, na Escola Municipal de Ensino Infantil e Fundamental Infante Paulo Sérgio Sousa Lira, no bairro Passaré. Foi examinada uma amostra de 186 alunos (59\%) dos 315 matriculados em 12 turmas, desde o Jardim I ao $5^{\circ}$ ano, nos turnos da manhã e da tarde.

Antes do exame de cavidade oral houve instrução de higiene oral e escovação supervisionada com dentifrícios fluoretados em todos os escolares examinados. Inicialmente foi demonstrada uma técnica de escovação simplificada em um macromodelo, para permitir melhor entendimento dos alunos. Em seguida foram distribuídos kits de higiene dental, contendo uma escova dental, um dentifrício e fio dental. 
Cada classe foi dividida em pequenos grupos de 3 a 4 alunos, para ir até o escovódromo da escola, para aplicar a técnica ensinada anteriormente.

Uma vez realizada a escovação supervisionada em cada aluno, foi executada a etapa do exame clínico em ambiente com luz natural. $\mathrm{O}$ exame foi realizado por uma examinadora, estudante de Odontologia do $6^{\circ}$ período, previamente capacitada e calibrada para executar o levantamento epidemiológico, supervisionada pelo cirurgião-dentista, profissional da Unidade Básica de Saúde.

Considerando que se está trabalhando com levantamento de necessidades, foi utilizado um formulário estruturado contendo o nome e a idade, e a identificação das seguintes alterações:

- 0 : sem necessidades

- 1: sangramento gengival

- 2: atividade de cárie até 3 cavidades/mancha branca

- 3: atividade de cárie 4 cavidades ou mais

- 4: raiz residual

Um terceiro momento consistiu na análise dos dados obtidos, para posteriormente encaminhar os escolares, que apresentam maior necessidade de tratamento, ou seja, atividade de cárie/mancha branca e presença de raiz residual, para atendimento odontológico na unidade de saúde, enquanto os demais alunos receberam instrução de higiene oral para reverter o quadro apresentado como no sangramento gengival.

Os princípios éticos foram seguidos em todas as fases do estudo, em consonância com o que preconiza a Resolução 196/96 do Conselho Nacional de Saúde. O presente projeto de pesquisa foi submetido ao Comitê de Ética em Pesquisas da Universidade de Fortaleza, sendo aprovado sob CAAE 0519.0.037.000-11. Os investigadores foram responsáveis por conduzir o estudo em restrita observação ao protocolo aprovado.

Os dados foram tabulados em planilhas do Excel $\AA$, e analisados através do software SPSS ${ }^{\circledR}$ 16. Serão apresentados através de médias e frequências.

\section{RESULTADOS}

O número total de alunos examinados foi de 186 alunos, sendo 104 $(55,9 \%)$ do sexo masculino e 82 do sexo feminino (44,1\%), da faixa etária de 4 a 15 anos. No contexto geral 55,4\% apresentaram alguma alteração na cavidade oral.

A classificação das necessidades da cavidade oral foi agrupada na Tabela 1 de acordo com o código, sua frequência e porcentagem. 
Tabela 1 - Classificação das necessidades bucais dos alunos da escola EMEF Infante Paulo Sérgio Sousa Lira, do bairro Passaré. Fortaleza, 2010.

\begin{tabular}{llll}
\hline & $\begin{array}{l}\text { Freqüência } \\
\text { escolares }\end{array}$ & $\begin{array}{c}\text { Pré Freqüência } \\
\text { em escolares }\end{array}$ & Total \\
\hline 0 & $17(56,6 \%)$ & $66(42,3 \%)$ & $83(44,6 \%)$ \\
1 & $0(0 \%)$ & $1(0,6 \%)$ & $1(0,5 \%)$ \\
2 & $6(20 \%)$ & $53(33,9 \%)$ & $59(31,7 \%)$ \\
3 & $6(20 \%)$ & $10(6,4 \%)$ & $16(8,6 \%)$ \\
4 & $1(3,3 \%)$ & $26(16,6 \%)$ & $27(14,5 \%)$ \\
Total & $30(100 \%)$ & $156(100 \%)$ & $186(100 \%)$ \\
\hline
\end{tabular}

Para que se pudesse analisar a existência de correlação entre a presença de alterações bucais e a faixa etária, os alunos também foram categorizados segundo a escolaridade (pré-escolares e escolares) e em indivíduos com ou sem alterações bucais (Tabela 2).

Tabela 2 - Distribuição do grau de escolaridade e ocorrência de alterações bucais

\begin{tabular}{llll}
\hline & Com alteração & Sem alteração & Total \\
\hline Pré-escolar & $13(43,4 \%)$ & $17(56,6 \%)$ & $30(16 \%)$ \\
Escolar & $90(57,6 \%)$ & $66(42,4 \%)$ & $156(84 \%)$ \\
Total & $103(55,4 \%)$ & $83(45 \%)$ & 186 \\
\hline
\end{tabular}

Como os dados são nominais, foi utilizado o Teste Exato de Fisher para averiguar a presença de significância estatística $(p<0,05)$. Não houve associação estatística significativa entre as variáveis.

\section{DISCUSSÃO}

Diversos estudos comprovam a importância da educação, em particular da mãe, na saúde bucal das crianças. Isso se dá tanto pelo reflexo na própria condição de adequação do meio bucal da mãe, que retardaria o contato da criança com microorganismos cariogênicos, como na implementação de cuidados de alimentação e higienização decorrentes de maior grau de informação ${ }^{16}$. Esta é uma variável que deve ser avaliada em estudos a posteriori.

Em idades diferentes na infância, os hábitos de escovação devem ser apresentados às crianças por seus pais ou cuidadores e praticados diariamente. Portanto, uma abordagem educativa direcionada tanto para crianças quanto para seus pais os ajudaria a sofrer um número menor de lesões de cáries e a apresentar saúde bucal e qualidade de vida melhores ${ }^{7}$.

Embora a maioria das mães (71\%) demonstra estar ciente da necessidade da escovação diária supervisionada, apenas cerca de $40 \%$ delas supervisiona a escovação dos filhos. Foi constatado também que apenas $40 \%$ das crianças cuidam da sua própria higiene bucal ${ }^{7}$.

Inúmeros estudos têm apontado para uma associação entre determinantes sócioeconômicos e a presença da cárie dentária, entre outros, demonstrando que as piores condições de saúde bucal não podem ser dissociadas, sobretudo, das disparidades de renda e do grau de escolaridade, fatores indicativos do nível social ${ }^{9}$.

Uma condição financeira precária pode acarretar prejuízos na saúde bucal, 
pois é necessário que cada membro da família possua sua própria escova dental, evitando assim, compartilhar só uma².

Apesar da maioria das crianças avaliadas apresentar alterações na cavidade bucal, quando comparamos com a prevalência de cárie do último censo brasileiro de saúde bucal, destaca-se o número de crianças da população estudada sem alterações ${ }^{4}$. Principalmente na idade pré-escolar. A média de higidez encontrada nos pré-escolares examinados supera em $10 \%$ a prevalência nacional, onde aos 5 anos de idade $46,6 \%$ das crianças brasileiras estão livres de cárie na dentição decídua $^{10}$.

Nos pré-escolares, deve-se considerar uma série de fatores que podem ocasionar a cárie dentária. É necessário que se tenha a presença de bactérias cariogênicas nos dentes, além de outros fatores que também são importantes. Destacam-se o tipo e a consistência do alimento ingerido pela criança, a frequência desta alimentação e a susceptibilidade da criança em apresentar a doença ${ }^{15}$.

O risco de cárie aumenta quando carboidratos fermentáveis são consumidos em alta frequência e/ou sob uma forma que permita sua retenção na cavidade bucal por longos períodos ${ }^{3}$.

A tendência a higidez bucal também acontece entre os escolares do referido colégio, que apresentam ausência de alterações bem acima da média da própria cidade de Fortaleza, que possui a média de dentes hígidos para a faixa etária de 5 anos de apenas $18 \%$, enquanto que a prevalência de cárie é de $86 \%{ }^{4}$. O número de escolares com dentição hígida pela média nacional e do município é de $43,5 \%$ e $25 \%$ respectivamente ${ }^{10}$.

$$
\text { Segundo Mondelli }{ }^{1} \text {, a anatomia }
$$
dentária dos elementos permanentes influencia no aparecimento da cárie, pois apresenta sulcos mais profundos e devido à progressão da doença, que já se encontra com cavidade profunda, leva a consequente perda dentária.

Segundo Traebert ${ }^{1}{ }^{8}$, os dados aqui relatados podem mostrar-se úteis para a definição de programas de prevenção e tratamento da doença, para o planejamento dos serviços. Elas são importantes, pois mostram a necessidade de implementar políticas sociais que priorizem as classes sociais mais carentes a fim de reduzir a quantidade de alterações bucais encontradas nas crianças com idade pré-escolar e escolar.

De acordo com Castilho ${ }^{7}$, a educação em saúde bucal e medidas preventivas na infância ajudam a reduzir a necessidade de intervenções cirúrgicas no futuro. Entretanto, os pais devem conseguir assimilar as informações e entender as instruções fornecidas e, então, implementá-las como parte da rotina preventiva de saúde bucal dos filhos.

\section{CONCLUSÕES}

O estudo periódico da incidência das alterações bucais em indivíduos em idade pré-escolar e escolar é muito importante para os municípios, pois ele vai orientar o planejamento e a avaliação das ações desenvolvidas.

Também é de fundamental importância o estreitamento do vínculo entre as ESF e as escolas tanto para o diagnóstico precoce, quanto para o tratamento das alterações detectadas.

Diante dos resultados obtidos pôdese constatar que existe elevada porcentagem de estudantes com alterações na cavidade bucal, principalmente a cárie dentária logo no início da infância. 
Portanto, é indispensável a realização de ações coletivas, incluindo-se a escovação supervisionada e as palestras educativas, a fim de se reverter esta realidade.

As ações curativas/restauradoras não devem, porém ser relegadas a segundo plano, visto que a doença cárie já se instalou em mais da metade da população examinada.

$\mathrm{O}$ inquérito de necessidades em saúde bucal orienta a coleta de dados para a posterior análise e tomada de decisões no planejamento da atenção individual e coletiva. É um instrumento de Vigilância Epidemiológica utilizado com a finalidade de planejamento das ações de saúde bucal, subsidiando $\mathrm{o}$ agendamento para $\mathrm{o}$ atendimento individual e orientando a frequência da participação.

Trata-se de um indicador individual que gera uma classificação coletiva. Possibilita uma configuração quantificada e qualificada das necessidades de atenção dos grupos, dividindo as necessidades da população em categorias de acordo com a complexidade e o tipo de recurso humano requerido nos procedimentos coletivos.

\section{REFERÊNCIAS}

1. Almeida GCM; Ferreira MAF. Saúde bucal no contexto do Programa Saúde da Família: práticas de prevenção orientadas ao indivíduo e ao coletivo. Cad. de Saúde Pública, Rio de Janeiro, 2008; 24(9):2131-40.

2. Baldani $\mathrm{MH}$; Vasconcelos AGG; Antunes JLF. Associação do índice CPO-D com indicadores. Caderno de Saúde Pública, Rio de Janeiro. 2004 Fev; 20(1):143-152.

3. Brandão IMG et al. Cárie precoce: influência de variáveis sócio- comportamentais e do locus de controle da saúde em um grupo de crianças de Araraquara, São Paulo, Brasil. Cad. Saúde Pública, Rio de Janeiro. 2006; 22(6): 1247-56

4. Brasil Projeto SBBrasil 2010: Pesquisa Nacional de Saúde Bucal - Resultados Principais. Secretaria de Atenção à Saúde/Secretaria de Vigilância em Saúde. Brasília. 2011.

5. Cadernos de Atenção Básica - Saúde na Escola. Ministério da Saúde. Brasília, 2009.

6. Carvalho MF et al. Correlação entre a merenda escolar, obesidade e cariogenicidade em escolares. Revista Odonto, São Bernardo do Campo. 2009; 34(17): 56-63.

7. Castilho ARF, Mialhe FL, Barbosa TS, Rontani RMP. Influência do ambiente familiar sobre a saúde bucal das crianças: uma revisão sistemática. Jornal de pediatria. Mar 2013. 89(2):116-23.

8. Cavalcante CAT et al. Levantamento de necessidades em saúde bucal e a organização da atenção para crianças de zero a seis anos de 166 creches de Belo Horizonte. 2005.

9. Fadel CB; Saliba NA. Aspectos sóciodentais e de representação social da cárie dentária no contexto maternoinfantil. RGO, Porto Alegre. 2009; 57(3): 303-309.

10. Ministério da Saúde, Secretaria de Atenção à Saúde, Política Nacional de Humanização da Atenção e Gestão do SUS. Acolhimento e classificação de risco nos serviços de urgência. Brasília: Ministério da Saúde, 2009.

11.Mondelli J. Fundamentos de Dentística Operatória. $1^{\circ}$ ed. São Paulo: Santos, 2006. 
12. Nickel DA; Lima FG; Silva BB. Modelos assistenciais em saúde bucal no Brasil. Cad. Saúde Pública, Rio de Janeiro. 2008; 24(2): 241-46.

13. Programa Saúde na Escola - Manual para adesão. Ministério da Saúde e Ministério da Educação, Brasília. 2013.

14. Sá LCA; Vasconcelos MMVB. Levantamento das necessidades odontológicas em crianças de 12 anos da zona urbana do município de Verdejante-PE. Odontologia. Clín.científ., Recife. 2008; 7(4): 325-29.

15. Saliba NA et al. A dieta escolar e a prevenção da cárie dentária nas escolas públicas. Revista Odontológica de Araçatuba. 2003; 24(2): 17-22.

16. Santos MF et al. Relação entre doença cárie e gengivite e condições socioeconômicas dos usuários da creche comunitária centro infantil murialdo. Boletim da Saúde, Porto Alegre. 2004; 18(1): 114-26.

17. Tuoni ACLF; Lacerda JT; Traebert J. Prevalência de Cárie em Escolares da Zona Rural de Jacinto. Pesquisa Brasileira em Odontopediatria e Clinica Integrada, Joâo Pessoa. 2007 Dez; 7(3): 277-84.

18. Traebert $\mathrm{J}$ et al. Prevalência e severidade de cárie dentária. Caderno de Saúde Pública, Rio de Janeiro. 2002 Mai; 18(3): 817-21.

19. Vilhena FV et al. Novo protocolo para as ações de saúde bucal coletiva:. Ciência \& Saúde Coletiva, São Paulo. 2008; 13(2): 2097-2103, 2008.

\section{AGRADECIMENTOS}

Gostaríamos de agradecer a Universidade de Fortaleza (UNIFOR)

\section{ABSTRACT \\ Organization of children's oral health by health in schools: Needs assessment}

The objective of this study was to survey the needs buccal of the students of Fortaleza in Passaré neighborhood. The study was cross-sectional in a public school. The first step consisted of oral hygiene instruction and supervised brushing with fluoridated toothpaste in all students. Then in an environment with natural light was conducted clinical examination. According to the characteristics of the oral cavity was filled in a form with the following codes: 0 - no needs, 1 - bleeding gums, 2 - caries activity up to 3 wells / white spot, 3 - caries activity up to 4 or more cavities and 4 - residual root. Data processing was performed in the SPSS ${ }^{\circledR}$ 16. We examined a total of 186 students, divided into 12 classes, from pre-school to school. Of all students, 55.4\% showed abnormalities mouth. Of preschool children, $43.4 \%$ had caries activity, bleeding or residual root while in school this frequency was $57.6 \%$. The prevalence found in this community is below the national and state prevalence. Inferring probably good preventive work of dentists this territory.

Descriptors: Health surveys. Epidemiology. Students. Oral health. 